Published in final edited form as:

Nat Genet. 2020 January ; 52(1): 29-34. doi:10.1038/s41588-019-0547-z.

\title{
Extrachromosomal circular DNA drives oncogenic genome remodeling in neuroblastoma
}

\author{
Richard P. Koche ${ }^{1,{ }^{*}}$, Elias Rodriguez-Fos ${ }^{2,{ }^{*}}$, Konstantin Helmsauer ${ }^{3,{ }^{*}}$, Martin Burkert ${ }^{4,5,8}$, \\ Ian C. MacArthur ${ }^{3}$, Jesper Maag ${ }^{1}$, Rocio Chamorro ${ }^{3}$, Natalia Munoz-Perez ${ }^{3}$, Montserrat \\ Puiggròs $^{2}$, Heathcliff Dorado Garcia ${ }^{3}$, Yi Bei ${ }^{3}$, Claudia Röefzaad ${ }^{3}$, Victor Bardinet ${ }^{3}$, \\ Annabell Szymansky ${ }^{3}$, Annika Winkler ${ }^{3}$, Theresa Thole ${ }^{3}$, Natalie Timme ${ }^{3}$, Katharina \\ Kasack $^{6}$, Steffen Fuchs ${ }^{3,5,6}$, Filippos Klironomos ${ }^{3}$, Nina Thiessen ${ }^{5}$, Eric Blanc ${ }^{5}$, Karin \\ Schmelz ${ }^{3}$, Annette Künkele ${ }^{3,5,6}$, Patrick Hundsdörfer ${ }^{3,5,6}$, Carolina Rosswog 7 , Jessica \\ Theissen $^{7}$, Dieter Beule ${ }^{5}$, Hedwig Deubzer ${ }^{3,6,10}$, Sascha Sauer ${ }^{5}$, Joern Toedling ${ }^{3}$, Matthias

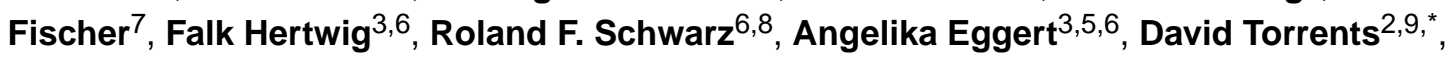 \\ Johannes H. Schulte ${ }^{3,5,6,{ }^{*}}$, Anton G. Henssen $3,5,6,10,{ }^{*}$ \\ ${ }^{1}$ Center for Epigenetics Research, Memorial Sloan Kettering Cancer Center, New York, New York, \\ USA.
}

${ }^{2}$ Barcelona Supercomputing Center (BSC), Joint BSC-CRG-IRB Research Program in Computational Biology, 08034, Barcelona, Spain

${ }^{3}$ Department of Pediatric Oncology/Hematology, Charité-Universitätsmedizin Berlin, Germany.

${ }^{4}$ Department of Biology, Humboldt University, Berlin, Germany

${ }^{5}$ Berlin Institute of Health, 10178 Berlin, Germany.

${ }^{6}$ German Cancer Consortium (DKTK), partner site Berlin, and German Cancer Research Center (DKFZ), Heidelberg, Germany.

${ }^{7}$ Department of Experimental Pediatric Oncology, University Children's Hospital of Cologne, and Center for Molecular Medicine Cologne (CMMC), Medical Faculty, University of Cologne, 50937 Cologne, Germany

${ }^{8}$ Max-Delbrück-Centrum für Molekulare Medizin (BIMSB/BIH), Berlin, Germany.

9Institució Catalana de Recerca i Estudis Avançats (ICREA), 08010, Barcelona, Spain.

\footnotetext{
Correspondence should be addressed to R.P.K. or A.G.H. (kocher@mskcc.org and henssenlab@ gmail.com). Author Contributions:

R.P.K., E.R.F., K.H., J.M., F.H., I.C.M., R.C., A.W., MB., M.P., Cl.R., T.T., H.D., R.S., Ca.R., J.Th., V.B., N.M.P., H.D.G., Y.B., A S., N.Ti., K.K., S.F., N.Th., E.B., K.S, A.K., P.H., J.To., M.F., D.B., S.S., A.E., D.T., J.H.S., A.G.H. contributed to the study design and collection and interpretation of the data. R.P.K. performed the analysis of Circle-seq and whole-genome sequencing. E.R.F. performed the data analysis of the whole-genome sequencing data. I.C.M., R.C., N.M.P., H.D.G. performed circular DNA extraction and PCRbased validation. V.B., Cl.R., A.W., P.H., K.S., M.F., A.S., F.H. collected and prepared patient samples. C.Ra., J.Th. performed and analyzed FISH. M.B. and R.S. performed haplotype phasing analyses. M.P. and J.To. analyzed tumor genome-sequencing data. M.B. performed allele-specific analysis of Circle-seq, whole-genome sequencing and RNA-seq. S.F., F.K., R.P.K, K.H. and J.M. performed RNA-seq data analysis. PH., H.D.G., N.M.P., A.S., D.B., K.S., performed experiments and analyzed data. R.P.K., A.K., A.E. and J.H.S. contributed to study design. R.P.K. and A.G.H. led the study design, performed data analysis and wrote the manuscript, to which all authors contributed.

* These authors contributed equally to this work.

Competing interests

The authors have no competing interests to declare.
} 
${ }^{10}$ Experimental and Clinical Research Center (ECRC) of the MDC and Charité Berlin, Berlin, Germany.

\section{Introductory paragraph}

Extrachromosomal circularization of DNA is an important genomic feature in cancer. The structure, composition and genome-wide frequency of extrachromosomal circular DNA, however, have not yet been extensively profiled. Here, we combined genomic and transcriptomic approaches to describe the landscape of extrachromosomal circular DNA in neuroblastoma, a tumor arising in childhood from primitive cells of the sympathetic nervous system. Our analysis identifies and characterizes a wide catalog of somatically acquired and undescribed extrachromosomal circular DNAs. Moreover, we find that extrachromosomal circular DNAs are an unanticipated major source of somatic rearrangements, contributing to oncogenic remodeling through chimeric circularization and reintegration of circular DNA into the linear genome. Cancercausing lesions can emerge out of circle-derived rearrangements and are associated with adverse clinical outcome. It is highly probable that circle-derived rearrangements represent an ongoing mutagenic process. Thus, extrachromosomal circular DNAs represent a multi-hit mutagenic process, with important functional and clinical implications for the origins of genomic remodeling in cancer.

Recent studies have shown that extrachromosomal circular DNA is more prevalent in human tissues than previously anticipated ${ }^{1-5}$. Based on size and copy number, at least three classes of circular DNA exist in human cells: i. small extrachromosomal circular DNA (here referred to as eccDNA, including microDNAs) ${ }^{3,6}$, ii. large, copy number amplified extrachromosomal ecDNA ${ }^{1}$, and iii. ring and/or neochromosomes ${ }^{7,8}$. It is commonly accepted that ecDNA can lead to oncogene amplification and is a powerful driver of intratumoral heterogeneity $1,9,10,11,12$. Whether extrachromosomal circular DNA has other cancer-causing functions is unknown, and the impact circularization has on genome remodeling is unclear. Neuroblastoma is one of the first tumor entities in which extrachromosomal oncogene circularization in the form of $M Y C N$ proto-oncogene double minute chromosomes was detected ${ }^{10,13}$. Since the first descriptions in $1965^{14,15}$, the extent of DNA circularization has not been accurately quantified in neuroblastoma. We hypothesized that extrachromosomal circular DNA could represent a genome-wide, driving mutagenic process in neuroblastoma with functional consequences beyond oncogene amplification. We set out to systematically describe the spectrum and impact of extrachromosomal circular DNA in neuroblastoma using different genomic and transcriptomic approaches (Supplementary Fig. 1).

Since DNA circularity can be computationally inferred from whole-genome sequencing (WGS) data ${ }^{3,16,17}$, we applied an algorithm utilizing paired-end read orientation to detect circularity to WGS from 93 neuroblastomas paired with normal blood specimens (Fig. 1a,b). This approach detected a large tumor-specific circular DNA catalog, including $M Y C N$ double minute chromosomes, mitochondrial DNA and many previously undescribed ecDNAs and eccDNAs (Fig. 1c,d; Supplementary Fig. 2a,b). This suggests a greater prevalence and complexity of extrachromosomal circular DNA in neuroblastoma than previously anticipated. To achieve complementary and more sensitive detection and 
characterization of extrachromosomal circular DNA in neuroblastoma, we adapted and modified the Circle-seq method (Supplementary Fig. 1, 2c, 2d) ${ }^{6}$. We achieved specific DNA circle enrichment through $>10^{10}$-fold depletion of linear genomic DNA (Fig. 1c, Supplementary Fig. 2c, 3a-c). Applying Circle-seq to endonuclease-treated genomic DNA significantly reduced reads mapping to circularized genomic regions by 474 fold $(P=7.566$ $\times 10^{-11}$, Welch's t-test, Fig. 1c; Supplementary Fig. 3d,e), confirming specific enrichment of circular DNA. Sequence composition was analyzed and genomic origin inferred combining massive parallel paired-end sequencing with long-read Nanopore and single molecule realtime sequencing. Circular head-to-tail junctions predicted computationally were confirmed by PCR and Sanger sequencing (Supplementary Fig. 3a-c). De novo sequence assembly of long reads spanning the entirety of circles allowed further physical confirmation of their circular structure in $60 \%$ of cases (Supplementary Fig. 4a-c). Circle-seq confirmed $100 \%$ of ecDNAs and $30 \%$ of eccDNAs predicted from WGS and identified on average 0.86 ecDNAs and 5,673 eccDNAs per neuroblastoma (Fig. 1c-e, Supplementary Fig. 4d-f). Although ecDNA was accurately predicted from WGS with high sensitivity (100\%), our results highlight the advantages of using additional and more sensitive approaches, such as Circleseq, to obtain a comprehensive characterization of extrachromosomal circular DNA in tumors.

The structure of circularized genomic loci in neuroblastoma varied considerably, with mean sizes of 680,200 bp (ecDNA) and 2,403 bp (eccDNA) in tumors, reproducing the oscillating length distribution observed in lymphoma cancer cell lines ${ }^{3}$ (Fig. 1f; Supplementary Fig. $4 \mathrm{~g}-\mathrm{i})$. Consistent with cytogenetic reports ${ }^{18}$, no ring chromosomes were detected in neuroblastoma. Notably, both ecDNAs and eccDNAs were of mono-allelic origin, as determined by haplotype phasing (Fig. 1g). Inspection of circle junction sequences (ecDNA and eccDNA) indicated probable mechanism(s) of generation, since $2.8 \%$ contained nontemplate insertions indicative of non-homologous end joining repair or replicationassociated mechanisms (Supplementary Fig. 4k). In line with reports in human lymphoma cell lines ${ }^{19}, 6.3 \%$ of circle junctions contained sequence microhomologies (minimally $5 \mathrm{bp}$ ), suggesting the involvement of microhomology-mediated DNA repair (Supplementary Fig. 41). Notably, eccDNA and ecDNA were significantly enriched in genic regions, particularly in $M Y C N$-amplified neuroblastomas (Fig. 1h, Supplementary Fig. 5a-c). Whereas ecDNAs regularly contained entire genes $(62.5 \%)$, eccDNAs mostly included fractions of genes (Fig. 1i). Our genome-wide map of extrachromosomal circular DNA in neuroblastoma shows that DNA circularization is not restricted to proto-oncogenes but also affects various coding and noncoding regions with yet unknown functional consequences.

Extrachromosomal circularization and amplification are associated with increased oncogene expression. It remains unclear whether circularization itself or subsequent circle copy number amplification drives overexpression. The majority of genomic amplifications $(85.7 \%)$ identified using WGS coincided with ecDNAs, as confirmed by Circle-seq, suggesting that ecDNAs contribute to genomic amplifications. Moreover, haplotype phasing showed that ecDNAs were exclusively derived from the amplified allele, confirming extrachromosomal circularization as a potential driver of high-level focal genomic amplifications (Fig. 2a,b). Notably, circle length was significantly associated with higher copy number of circularized regions (Supplementary Fig. $5 \mathrm{~d}, P<1 \times 10^{-4}$ ), implicating 
circle length as a determining factor for subsequent amplification/propagation of extrachromosomal circular DNA (Supplementary Fig. 5d-f). Consistent with its prominent role in neuroblastoma genesis, $M Y C N$ was the most recurrently extrachromosomally amplified and overexpressed gene in our cohort (Fig. 2b-e, Supplementary Fig. 5a-c). Other cancer-related genes listed in the COSMIC database ${ }^{20}$, were also circularized in tumors and neuroblastoma cell lines, including the JUN and MDM2 proto-oncogenes and SRY-box 11 (SOX11) and TAL2 transcription factors (Fig. 2c, Supplementary Fig. 5a-c). Genomic copy number of oncogenes contained in the majority of eccDNAs, however, was not altered (Supplementary Fig. 5g,h), suggesting that extrachromosomal circularization may be required but insufficient for oncogene amplification.

To determine the consequences of DNA circularization for gene expression, we performed total RNA sequencing on our neuroblastoma cohort. Whereas differences in gene expression were not observed for most genes affected by circularization in the form of small eccDNA (Fig. 2d, Supplementary Fig. 5i-j), massive increases in expression occurred for a small subset of genes entirely incorporated on circularized DNA and amplified as ecDNA (Fig. 2df). For example, neurotrophin 3 (NTF3), a neurotrophic factor with known importance in neuroblastoma ${ }^{21}$, was strongly expressed from amplified ecDNA (Fig. 2f). Allele-specific mRNA expression (ASE) analysis confirmed that increased gene expression originated from the circular allele (Fig. 2a,b). In contrast, ASE from copy number neutral extrachromosomal circles did not differ from non-circular counterparts (Supplementary Fig. 5g,i,j, binomial test for equal probability, $P=0.24$ ), suggesting that extrachromosomal DNA circularization was insufficient to induce high-level gene expression. Thus, even though extrachromosomal DNA circularization is a major route to gene amplification, it appears insufficient alone (without combined amplification) to increase gene expression. Given this observation, we hypothesized that extrachromosomal circular DNA may have additional, cancer-relevant functions.

The genome-wide frequency and functional impact of circle-derived structural rearrangements, such as chimeric circle formation (circular DNAs including parts from different chromosomes) ${ }^{17,22}$, and circular DNA re-integration ${ }^{23}$, in neuroblastomas are currently unknown. We hypothesized that beyond their ability to drive gene amplification, extrachromosomal circular DNA may serve as substrates for oncogenic genome remodeling. We sought evidence of genomic rearrangements at circularization loci (ecDNA and eccDNA) in WGS data (Supplementary Fig. 1). Strikingly, most intra- and interchromosomal rearrangements detected in neuroblastoma genomes coincided with regions of extrachromosomal circularization, supporting the idea of circle-mediated genome remodeling (Supplementary Fig. 6a,b). Visual inspection of Circos plots from each tumor showed that inter-chromosomal rearrangements at circularization loci often formed a treeshaped pattern, defined as clusters of at least three inter-chromosomal rearrangements with the same origin and branches reaching other distant genomic regions (Fig. 3a,b; Supplementary Fig. 7a-i). Tree-shaped rearrangement cluster origins significantly overlapped with ecDNAs, with hot spots on chromosomes 2 (including MYCN) and 12 (Fig. 3c, Supplementary Fig. 7i). Only 10.5\% of $M Y C N$-amplified neuroblastomas displayed homogenously staining regions (HSRs, Supplementary Table 1), consistent with their rarity in neuroblastomas ${ }^{14,24,25}$. Thus, the majority of $M Y C N$-derived tree-shaped rearrangements 
did not represent HSRs. Tree-shaped rearrangement patterns indicative of circle-derived rearrangements were detected in $9 \%$ of pediatric tumors in the analysis of an independent dataset of structural rearrangements in 546 pediatric cancer genomes ${ }^{26}$, confirming this pattern is neither entity-specific nor dependent on variant detection methods (Supplementary Fig. 7j). Our data reveal an unanticipated association between extrachromosomal circular DNA and somatic genomic rearrangements in neuroblastoma.

We reasoned that circle-derived tree-shaped rearrangements could either represent chromosomal circle integrations or formation of chimeric circles, incorporating different chromosomal parts. To test this, we inspected the rearrangement recipient sites for signs of extrachromosomal circularization and integration and performed de novo assembly of circular DNAs (ecDNA and eccDNA). Extrachromosomal circular DNAs (identified using Circle-seq) appeared in 5.5\% of rearrangement recipient sites (tree branch intercepts), indicating chimeric circle formation (Supplementary Fig. 6). This was confirmed by longread Nanopore sequencing and assembly-based circle reconstruction, determining chimeric structures in $2.1 \%$ of eccDNAs and $84 \%$ of ecDNAs with on average 2.2 and 4.8 chimeric segments, respectively. Chromosomal circle integration was defined as inter-chromosomal rearrangements connecting extrachromosomal circles with intrachromosomal sites (i.e. not detected by Circle-seq). The majority of rearrangement recipient sites (83.3\%) were classified as circle integrations (Fig. 3d, Supplementary Fig. 6), which were validated by visual inspection of split reads, allele-specific PCR and Sanger sequencing (Fig. 3d, Supplementary Fig. 8). Phased heterozygous SNPs near integration breakpoints further confirmed extrachromosomal DNA circles as the origin of integrations (Fig. 3d). Thus, circle-derived tree-shaped rearrangement clusters represent i. formation of chimeric circles and ii. chromosomal circle integrations.

To test the functional impact of circle-derived, tree-shaped rearrangements in neuroblastoma, we inspected rearrangement recipient sites for the presence of cancerrelevant genes and changes in gene expression (Fig. 4a). Circle integration sites and sites included in chimeric circles were significantly enriched for cancer relevant genes $(P=$ $0.033)$ and particularly for tumor suppressor genes $(P=0.033)$, whose expression varied from tumors where the same gene was not involved in circle-derived rearrangements (Fig. 4b,c; Supplementary Fig. 9). For example, integration of an extrachromosomal circle fragment into the $D C L K 1$ gene (shown in Fig. 3d) led to loss of heterozygosity and was associated with significant repression of $D C L K 1$ expression (Fig. 4b). Consistent with a tumor suppressor function in neuroblastoma, low DCLK1 expression was associated with adverse patient prognosis and shRNA-mediated DCLK1 knock-down significantly increased clonogenicity in neuroblastoma cell lines (Supplementary Fig. 10a-i). Notably, circle integration also occurred in proximity to the telomerase reverse transcriptase gene (TERT) and was associated with enhanced TERT expression (Fig. 4c). It is tempting to speculate that enhancer hijacking ${ }^{27}$ or disruption of other cis-regulatory elements could explain such expression changes. Chimeric circle formation, on the other hand, often resulted in simultaneous amplification of multiple proto-oncogenes and aberrant circle-specific fusion transcript expression in a subset of cases (Supplementary Fig. 11). Thus, circle-derived rearrangements can contribute to aberrant expression of cellular tumor suppressors and proto-oncogenes. 
Seemingly genetically identical $M Y C N$-amplified neuroblastomas can produce strong clinical heterogeneity, representing a conundrum in the field. We hypothesized that circlederived oncogenic lesions could functionally cooperate with extrachromosomal circular $M Y C N$ amplification, explaining some of the clinical heterogeneity observed. Indeed, the presence of circle-derived rearrangements was associated with adverse patient outcome (Fig. 4d). In line with our hypothesis, patients with $M Y C N$-amplified neuroblastomas and circlederived rearrangement clusters involving $M Y C N$ had significantly worse overall survival compared to patients with $M Y C N$-amplified tumors lacking such rearrangements (Fig. 4e). Contrastingly, the number of rearrangements in $M Y C N$-amplified tumors did not correlate with survival (Supplementary Fig. 12a-c). This implicates circle-derived rearrangements as clinically relevant genomic alterations in neuroblastoma.

Our work provides a comprehensive map of extrachromosomal DNA circularization in neuroblastoma, revealing this mutagenic process to be more frequent than previously anticipated. We demonstrate that the majority of genomic rearrangements in neuroblastoma involve extrachromosomal circular DNA, challenging our current understanding about cancer genome remodeling. Such rearrangements have previously gone largely undetected or underestimated in WGS analyses because integrative, sequencing-based methods identifying extrachromosomal circular DNA in tumor samples were lacking. In contrast to previous cytogenetic reports describing HSR-based circle integration and chimeric circle formation as a means of stable gene amplification, we conclude that extrachromosomally circularized DNA can actively contribute to genome remodeling with important functional and clinical consequences (Fig. 4f). It is tempting to speculate that factors exist, such as recently described oncogenic transposases ${ }^{28-30}$, that could induce a mutator phenotype in the presence of extrachromosomal circular DNA, driving circle-mediated genome remodeling. We envision that our findings extend to other cancers and that further detailed analyzes of circle-derived rearrangements will shed new insights into our understanding of cancer genome remodeling.

\section{Supplementary Material}

Refer to Web version on PubMed Central for supplementary material.

\section{Acknowledgments}

We are grateful to Alex Kentsis, Scott Armstrong, Birgitte Regenberg, Frank Speleman, Sven Perner, Uwe Ohler and Nicole Hübener for critical discussions, and Kathy Astrahantseff for editorial advice. We thank Daniele Sunaga-Franze, Claudia Quedenau, Madlen Sohn, Kirsten Richter and Claudia Langnick for technical support. A.G.H. is supported by the Deutsche Forschungsgemeinschaft (DFG, German Research Foundation) - 398299703 and the Wilhelm Sander Stiftung. A.G.H., A.K. and S.F. are participants in the BIH-Charité Clinical Scientist Program funded by the Charité - Universitätsmedizin Berlin and the Berlin Institute of Health. A.G.H., S.F., K.H. and V.B. are supported by Berliner Krebsgesellschaft e.V. K.H. is supported by Boehringer Ingelheim Fonds. This work was also supported by the TransTumVar project - PN013600. This project was supported by the Berlin Institute of Health (BIH) within the collaborative research project CRG04-TERMINATE-NB. We thank the patients and their parents for granting access to the tumor specimen and clinical information that were analyzed in this study. We thank B. Hero, H. Düren, N. Hemstedt of the Neuroblastoma Biobank and Neuroblastoma Trial Registry (University Children's Hospital Cologne) of the German Society of Pediatric Oncology and Hematology (GPOH) for providing samples and clinical data. 


\section{References}

1. Turner KM et al. Extrachromosomal oncogene amplification drives tumour evolution and genetic heterogeneity. Nature 543, 122-125, doi:10.1038/nature21356 (2017). [PubMed: 28178237]

2. Moller HD et al. Circular DNA elements of chromosomal origin are common in healthy human somatic tissue. Nat Commun 9, 1069, doi:10.1038/s41467-018-03369-8 (2018). [PubMed: 29540679]

3. Shibata Y. et al. Extrachromosomal microDNAs and chromosomal microdeletions in normal tissues. Science 336, 82-86, doi:10.1126/science.1213307 (2012). [PubMed: 22403181]

4. Pennisi E. Circular DNA throws biologists for a loop. Science 356, 996, doi:10.1126/science. 356.6342.996 (2017). [PubMed: 28596318]

5. Verhaak RGW, Bafna V. \& Mischel PS Extrachromosomal oncogene amplification in tumour pathogenesis and evolution. Nat Rev Cancer, doi:10.1038/s41568-019-0128-6 (2019).

6. Moller HD, Parsons L, Jorgensen TS, Botstein D. \& Regenberg B. Extrachromosomal circular DNA is common in yeast. Proc Natl Acad Sci U S A 112, E3114-3122, doi:10.1073/pnas. 1508825112 (2015).

7. TJIO JH \& LEVAN A. The chromosome number of a man. Hereditas 42, 1-6, doi:10.1111/j. 1601-5223.1956.tb03010.x (1956).

8. Garsed DW et al. The architecture and evolution of cancer neochromosomes. Cancer Cell 26, 653667, doi:10.1016/j.ccell.2014.09.010 (2014). [PubMed: 25517748]

9. Rausch T. et al. Genome sequencing of pediatric medulloblastoma links catastrophic DNA rearrangements with TP53 mutations. Cell 148, 59-71, doi:10.1016/j.cell.2011.12.013 (2012). [PubMed: 22265402]

10. Kohl NE et al. Transposition and amplification of oncogene-related sequences in human neuroblastomas. Cell 35, 359-367 (1983). [PubMed: 6197179]

11. deCarvalho AC et al. Discordant inheritance of chromosomal and extrachromosomal DNA elements contributes to dynamic disease evolution in glioblastoma. Nat Genet 50, 708-717, doi: 10.1038/s41588-018-0105-0 (2018). [PubMed: 29686388]

12. Nikolaev S. et al. Extrachromosomal driver mutations in glioblastoma and low-grade glioma. Nat Commun 5, 5690, doi:10.1038/ncomms6690 (2014). [PubMed: 25471132]

13. Schwab M. et al. Amplified DNA with limited homology to myc cellular oncogene is shared by human neuroblastoma cell lines and a neuroblastoma tumour. Nature 305, 245-248 (1983). [PubMed: 6888561]

14. Balaban-Malenbaum G. \& Gilbert F. Double minute chromosomes and the homogeneously staining regions in chromosomes of a human neuroblastoma cell line. Science 198, 739-741 (1977). [PubMed: 71759]

15. Cox D, Yuncken C. \& Spriggs AI Minute Chromatin Bodies in Malignant Tumours of Childhood. Lancet 1, 55-58 (1965). [PubMed: 14304929]

16. Sanborn JZ et al. Double minute chromosomes in glioblastoma multiforme are revealed by precise reconstruction of oncogenic amplicons. Cancer Res 73, 6036-6045, doi: 10.1158/0008-5472.CAN-13-0186 (2013). [PubMed: 23940299]

17. Deshpande V. et al. Exploring the landscape of focal amplifications in cancer using AmpliconArchitect. Nat Commun 10, 392, doi:10.1038/s41467-018-08200-y (2019). [PubMed: 30674876]

18. Avet-Loiseau H. et al. Morphologic and molecular cytogenetics in neuroblastoma. Cancer 75 , 1694-1699, doi:10.1002/1097-0142(19950401)75:7<1694::aid-cncr2820750721>3.0.co;2-z (1995). [PubMed: 8826929]

19. Dillon LW et al. Production of Extrachromosomal MicroDNAs Is Linked to Mismatch Repair Pathways and Transcriptional Activity. Cell Rep 11, 1749-1759, doi:10.1016/j.celrep.2015.05.020 (2015). [PubMed: 26051933]

20. Forbes SA et al. COSMIC: somatic cancer genetics at high-resolution. Nucleic Acids Res 45, D777-D783, doi:10.1093/nar/gkw1121 (2017). [PubMed: 27899578] 
21. Bouzas-Rodriguez J. et al. Neurotrophin-3 production promotes human neuroblastoma cell survival by inhibiting TrkC-induced apoptosis. J Clin Invest 120, 850-858, doi:10.1172/JCI41013 (2010). [PubMed: 20160348]

22. Xu K. et al. Structure and evolution of double minutes in diagnosis and relapse brain tumors. Acta Neuropathol, doi:10.1007/s00401-018-1912-1 (2018).

23. Storlazzi CT et al. Gene amplification as double minutes or homogeneously staining regions in solid tumors: origin and structure. Genome Res 20, 1198-1206, doi:10.1101/gr.106252.110 (2010). [PubMed: 20631050]

24. Villamon E. et al. Genetic instability and intratumoral heterogeneity in neuroblastoma with MYCN amplification plus 11q deletion. PLoS One 8, e53740, doi:10.1371/journal.pone.0053740 (2013).

25. Marrano P, Irwin MS \& Thorner PS Heterogeneity of MYCN amplification in neuroblastoma at diagnosis, treatment, relapse, and metastasis. Genes Chromosomes Cancer 56, 28-41, doi:10.1002/ gcc.22398 (2017). [PubMed: 27465929]

26. Grobner $\mathrm{SN}$ et al. The landscape of genomic alterations across childhood cancers. Nature 555, 321-327, doi:10.1038/nature25480 (2018). [PubMed: 29489754]

27. Northcott PA et al. Enhancer hijacking activates GFI1 family oncogenes in medulloblastoma. Nature 511, 428-434, doi:10.1038/nature13379 (2014). [PubMed: 25043047]

28. Henssen AG et al. PGBD5 promotes site-specific oncogenic mutations in human tumors. Nat Genet 49, 1005-1014, doi:10.1038/ng.3866 (2017). [PubMed: 28504702]

29. Henssen AG et al. Genomic DNA transposition induced by human PGBD5. Elife 4, doi:10.7554/ eLife.10565 (2015).

30. Henssen AG et al. Forward genetic screen of human transposase genomic rearrangements BMC Genomics 17, 548 (2016). [PubMed: 27491780] 

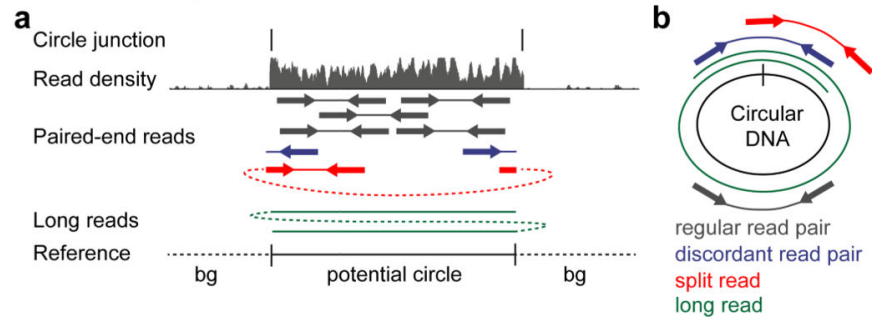

d

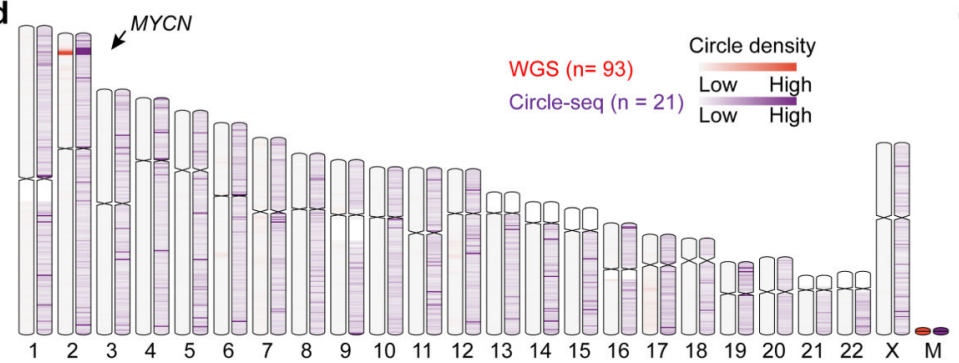

g

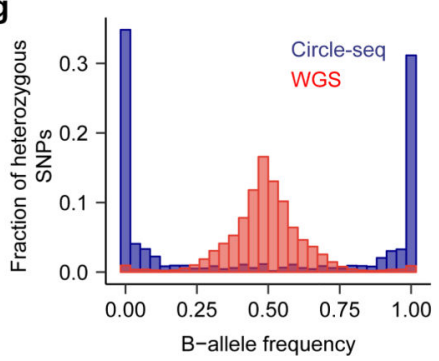

b

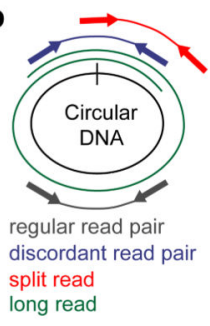

WGS

C

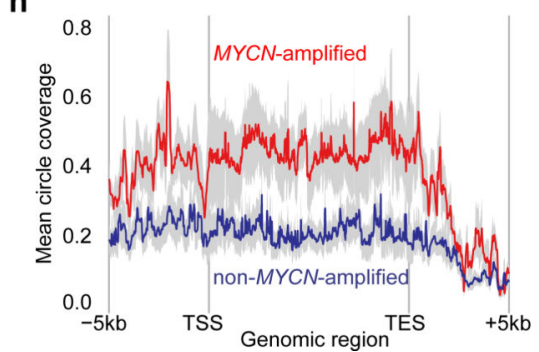

Circle-seq (SMRT)

Circle-seq (ILM)

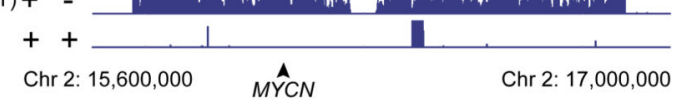

f

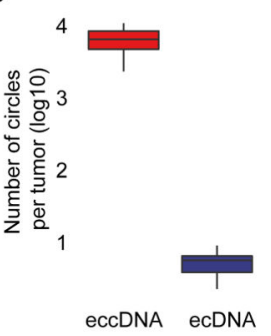

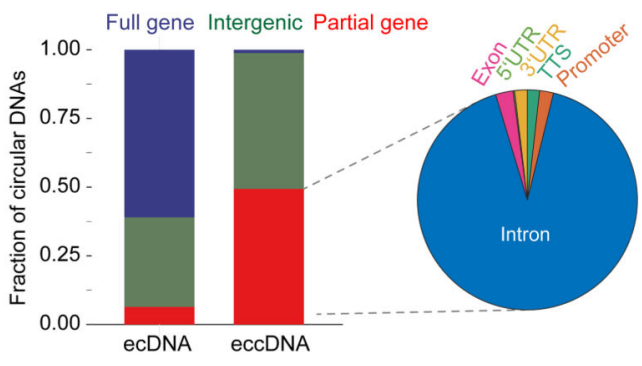

Fig. 1. A genome-wide map of extrachromosomal circular DNAs in neuroblastoma.

a, Schematic representation of sequencing reads as predicted for circular genomic regions (bg = background/non-circular genome). b, Schematic representation of sequencing read positions on extrachromosomal circular DNA. c, Genome tracks comparing sequencing read densities on an ecDNA as detected via WGS (only circle-specific head-to-tail reads are depicted), Circle-seq followed by paired-end sequencing (ILM) and single molecule realtime sequencing (SMRT) in neuroblastoma cells. DNA digestion with an exonuclease and/or endonuclease is indicated (+/-). Dotted blue line indicates predicted circle junction. Interruption of read density profile is due to lack of read alignment. (y - axis: 0-30 reads). d, Chromosome ideogram with genome-wide somatic extrachromosomal circular DNA density as inferred from WGS (blue) compared to Circle-seq (red; M, circular mitochondrial DNA).

e, Number of ecDNAs and eccDNAs per neuroblastoma ( $N=21$ tumors, $N=96,436$ eccDNAs, $N=14$ ecDNAs). f, Size distribution of ecDNAs and eccDNAs identified using Circle-seq in neuroblastomas ( $N=21$ tumors, $N=96,436$ eccDNAs, $N=14$ ecDNAs). g, Alternative-B-allele frequencies (BAF) in sequencing reads from Circle-Seq ( $N=21$ tumors) and WGS ( $N=93$ tumors). $\mathbf{h}$, Density of extrachromosomal circular DNA detected using Circle-seq over genic compared to gene-surrounding regions in $M Y C N$-amplified and nonamplified neuroblastomas ( $N=7 M Y C N$-amplified tumors, $N=14$ non-amplified tumors, lines represent mean signal and the shaded area represents the standard error of the mean). $\mathbf{i}$, Fraction of genomic regions affected by eccDNA compared to ecDNA ( $N=21$ tumors). TSS, transcription start site; TES, transcription end site. 
a

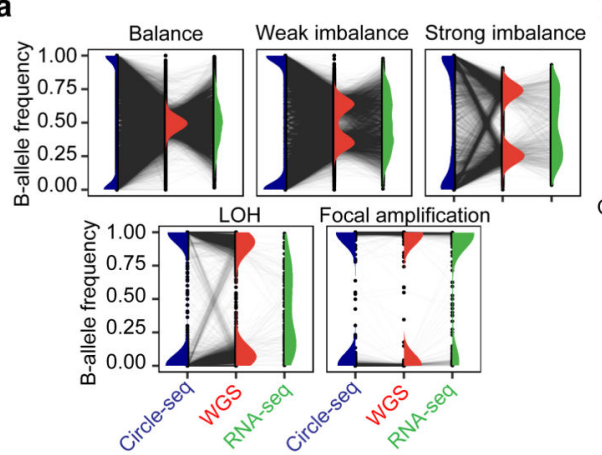

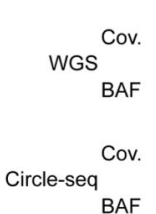

RNA-seq Cov. $\left.{ }^{9,000}\right]$

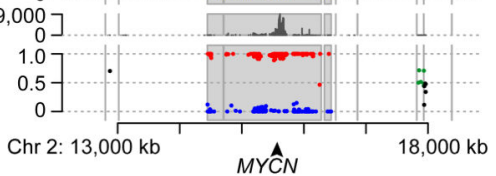

\section{d}

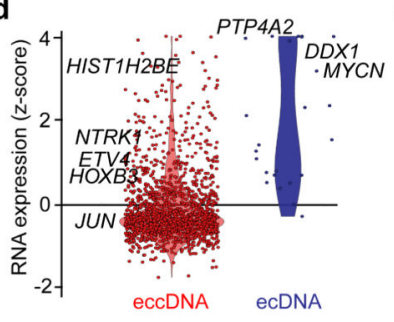

e

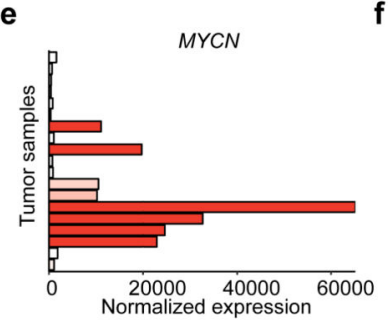

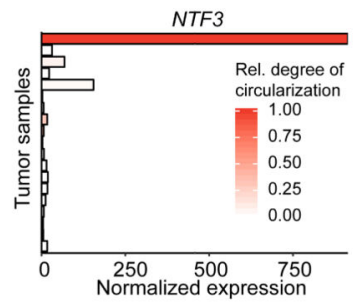

C

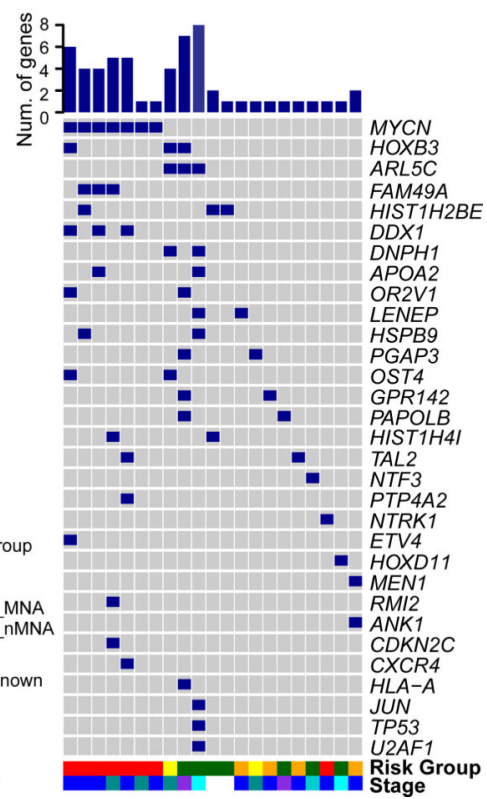

Fig. 2. Mono-allelic large extrachromosomal circular DNA are an origin of oncogene amplification and overexpression in neuroblastoma.

a, B-allele frequency of all extrachromosomal circular DNAs involving genes (both ecDNA and eccDNA) detected using Circle-seq (blue) compared to the corresponding genomic loci in whole-genome sequencing (red) and mRNA expressed from genes affected by DNA circularization measured using RNA sequencing (green, grey lines indicate corresponding measurements from Circle-seq, WGS and RNAseq, $N=18$ tumors). b, Genome track with phased reads from whole-genome sequencing of NB2013 (WGS), Circle-seq and RNA sequencing (RNA-seq) at the region of extrachromosomal circularization on chromosome 2 affecting $M Y C N$. (BAF= B-allele frequency, blue and red colored dots represent reads from different haplotypes) c, Genes (rows) affected by circularization in neuroblastoma samples (columns) as detected using Circle-seq ( $N=21$ tumors). d, Relative mRNA expression (zscores) of genes affected by extrachromosomal DNA circularization in the form of eccDNA $(N=1,696)$ compared to ecDNA $(N=24)$ as measured using total RNA sequencing $(N=21$ tumors). Normalized gene expression (mRNA) for $M Y C N$ proto-oncogene, bHLH transcription factor $(M Y C N, \mathbf{e})$ and neurotrophin $3(N T F 3, \mathbf{f})$ in neuroblastomas. The degree of gene circularization is indicated in red (see color scale). 
a

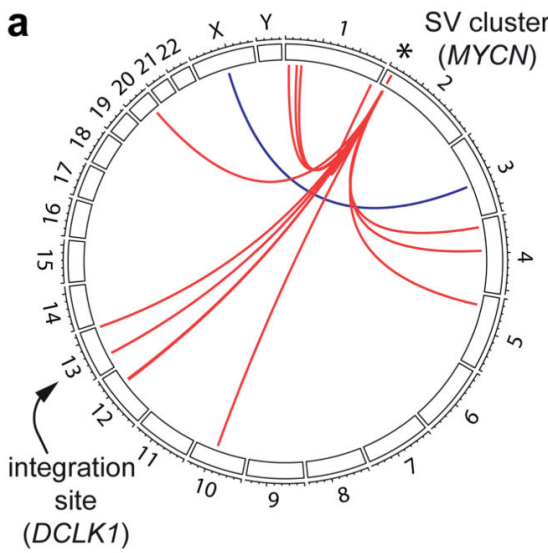

C

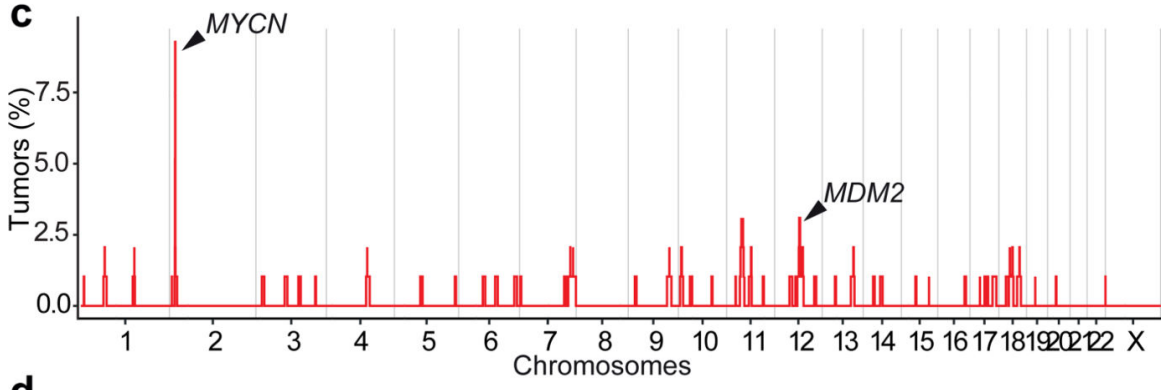

d

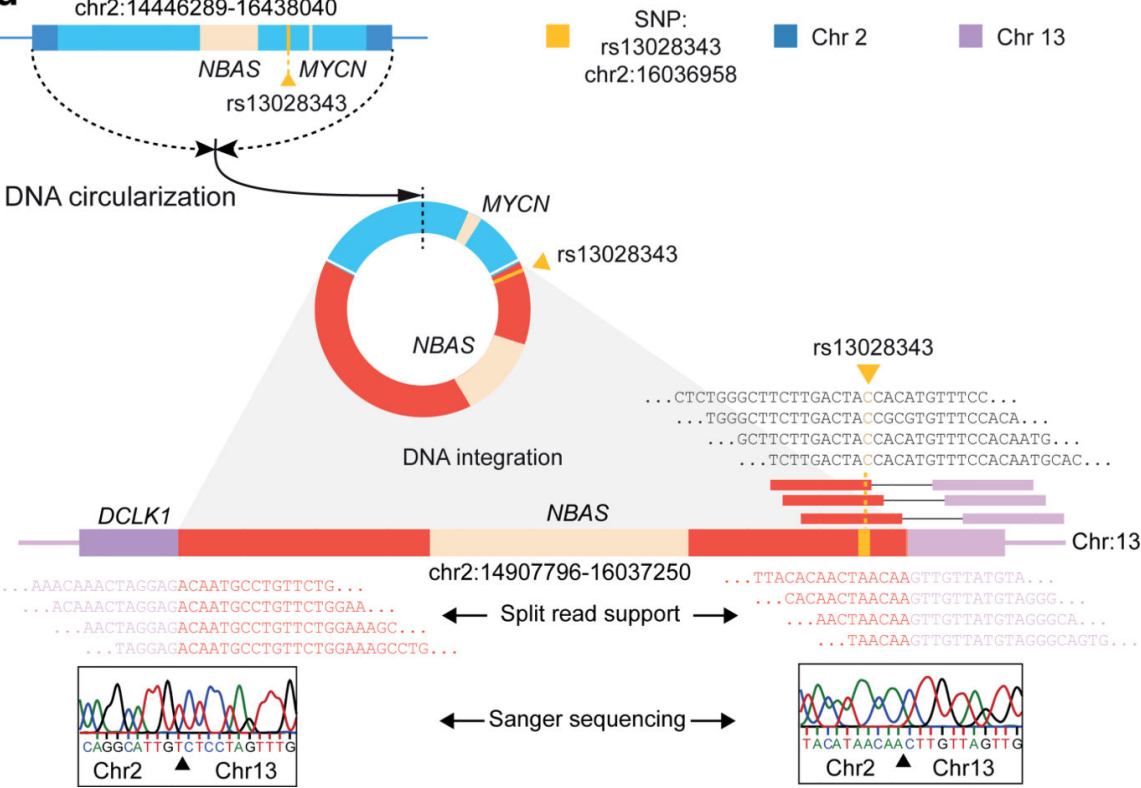

Fig. 3. The majority of structural rearrangements involve sites of extrachromosomal DNA circularization and form clustered rearrangement patterns in neuroblastoma.

a, Circos plot of inter-chromosomal rearrangements identified using five variant detection algorithms in one neuroblastoma genome (CB2013), shown exemplarily. Tree-shaped clustered rearrangement pattern (red), originating at a region of $M Y C N$ circularization (asterisk) is highlighted. b, Detailed view of genomic breakpoint localizations (black) at the base of the tree-shaped rearrangement cluster for the neuroblastoma shown in (a) define a region of clustered breakpoints (yellow) and overlaps with the region of extrachromosomal

Nat Genet. Author manuscript; available in PMC 2020 July 01. 
DNA circularization, as detected using Circle-seq (pink) and whole-genome sequencing (WGS, green). Copy number changes are highlighted in red. c, Genome-wide frequency of tree-shaped clusters of rearrangements in 93 primary neuroblastoma samples. The pattern is recurrently identified on chr2 (at the MYCNlocus), chr11 and chr12 (at the MDM2 locus). d, Schematic representation of circle integration in one exemplary neuroblastoma (CB2013). Genomic region, including $M Y C N$ (blue), is circularized, and parts of the extrachromosomal circle are integrated (red) into chromosome 13 (pink) leading to a disruption of DCLK1. Sequencing reads supporting a circle-specific SNP as well as split reads supporting circle integration are shown below. Sanger sequencing of integration breakpoints are shown in boxes. 
a

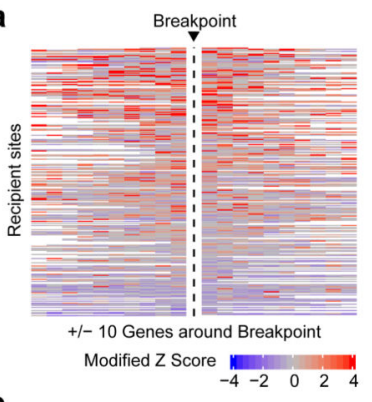

e

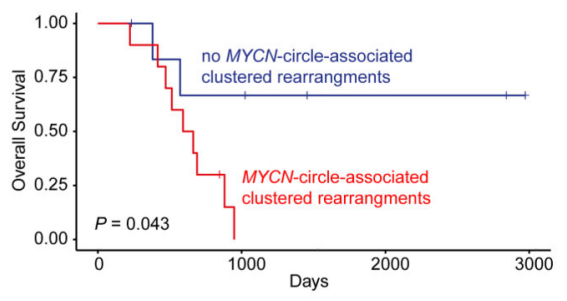

c

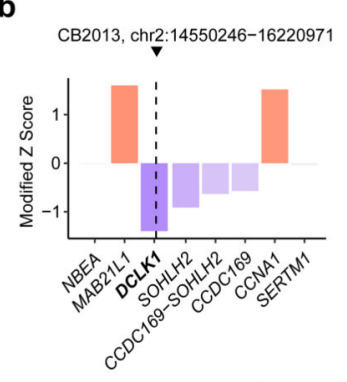

c

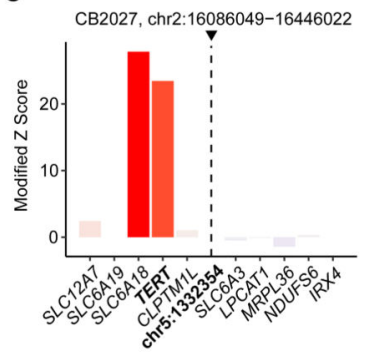

d

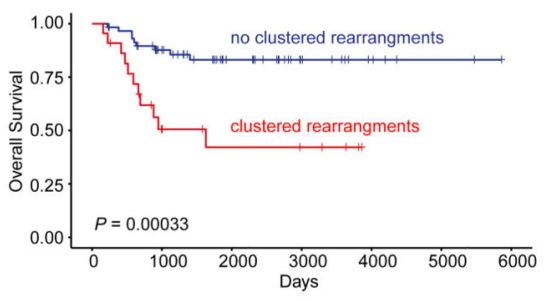

Fig. 4. Rearrangement of extrachromosomal circular DNAs drives transcriptional deregulation and dismal prognosis in neuroblastoma.

a, Heatmap showing differential expression of up to 10 genes located both up- and downstream or a maximal distance of $2 \mathrm{Mb}$ from each circle-derived rearrangement breakpoint ( $N=259$ breakpoints, $N=24$ tumors). Modified z-scores for the expression of cancer-relevant genes $D C L K 1$ (b) and TERT (c) affected by circle-derived rearrangements are shown for two representative genomic loci (in two neuroblastomas). d, Kaplan Meier analysis comparing neuroblastoma patient survival of patients with neuroblastomas affected by circle-derived clustered rearrangements ( $N=22$ patients) to patients with tumors lacking such rearrangements ( $N=59$ patients, $P=0.00033$ two-sided log-rank test). e, Kaplan Meier analysis comparing neuroblastoma patient survival with $M Y C N$-amplified tumors affected by $M Y C N$-circle-derived clustered rearrangements $(N=10)$ to patients with tumors lacking such rearrangements $(N=7, P=0.043$ two-sided log-rank test). f, Schematic diagram of the proposed mechanism of circle-mediated genome remodeling. 